\title{
ENTY - A ROMANIAN SYSTEM FOR THE OBJECTIVE EVALUATION OF BALANCE IN HUMANS
}

\author{
Mădălina GEORGESCU ${ }^{1}$, Daniel ROSNER ${ }^{2}$, Cristian ALEXANDRESCU ${ }^{2}$, \\ Flavia OPREA ${ }^{2}$, Andrei OSMAN ${ }^{3}$ \\ 1 "Carol Davila" University of Medicine and Pharmacy, Bucharest, Romania \\ 2 "Politehnica" University, Bucharest, Romania \\ ${ }^{3}$ University of Medicine and Pharmacy, Craiova, Romania \\ *Corresponding author: madalina.georgescu@gecad.com
}

DOI: $10.35189 /$ iphm.icpesk.2019.11

\begin{abstract}
Dizziness is one of the most frequent complaints in the general population, with a lifetime prevalence of about 20 to $30 \%$. Its long-term effects include imbalance and walking difficulties, disturbances which are only subjectively evaluated in the present by physicians. ENT physicians, neurologists, rehabilitation personnel and work physicians are all in contact with dizzy patients. Quantification of these deficits will improve assessment protocols for all physicians involved in treating this pathology, as well as monitoring the patient's progress under different treatment options. ENTy is a new, original Romanian medical device, based on an original methodology of measuring the patient's swaying during stance and the stepping trajectory. It identifies the movements during the two common vestibular tests, the Romberg and Unterberger tests, with the aid of an integrated inertial measurement unit. This is composed by an accelerometer, a gyroscope and a magnetometer. ENTy is a wearable wireless device, with a friendly interface designed to add value to the medical evaluation. It provides an output format ready for integration with medical records, data recording and automated data logging with available statistics and long-term follow-up options for a patient's evolution, both for medical progress tracking and for records auditing for workrelated medical check-ups. Currently, the report format is based on the existing normative guidelines for balance testing.
\end{abstract}

Keywords: stance and walking impairment, ENTy, original wearable wireless equipment, vestibular objective evaluation.

\section{Introduction}

Dizziness is one of the most frequent complaints in the general population. The lifetime prevalence of dizziness ranges from 20 to $30 \%$ (Neuhauser, 2007). ENT physicians, neurologists, rehabilitation personnel and labour physicians are all in contact with patients with dizziness and imbalance. These professionals all share the same goal - to ensure their patients have good quality of life, the ability to perform their daily activities independently and are able to return to their jobs. The key to the correct diagnosis and the differential diagnosis between central and peripheral vertigo and dizziness lays in patient history and the bedside examination even without laboratory tests (Cnyrim, Newman-Toker, Karch, Brandt, \& Strupp, 2008). Clinical examination together with vestibular tests gives a more accurate overview regarding a patient's stance, walking skills and dizziness or vertigo symptoms (Georgescu \& Stoian, 2011; Georgescu, Stoian, Mogoanță, \& Ciubotaru, 2012).

More important, objective vestibular investigations allow clinicians to quantify and monitor the vestibular impairment as well as the patient's evolution under appropriate management (Georgescu, 2017).

Otoneurological examination is mandatory for patients with dizziness and it usually includes nystagmus evaluation, as well as assessment of stance and walking performance.

Exploration of the vestibular function is a complex process, considering it is necessary to investigate the two important reflexes underlying the vestibular function - the vestibulo-ocular reflex (VOR) and the vestibulo-spinal reflex (VSR) (Abramovich \& Prasher, 1986).

The vestibule-spinal reflex (Figure 1) represents the connection between the vestibular receptors and nuclei and effector muscle groups (from the neck, trunk and lower limbs) involved in maintaining a stable body position.

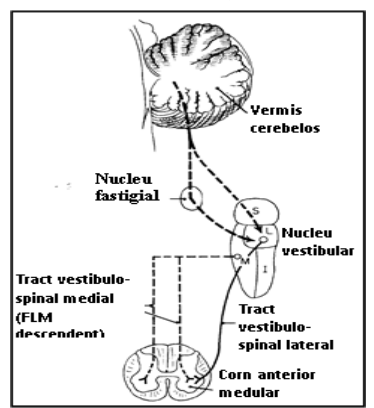

Figure 1. Vestibulo-spinal reflex 
The Romberg test/gait analysis performed with the eyes open and closed may indicate a sensory deficit, either vestibular or somatosensory.

The latter are most widely assessed clinically and the results are subjected to the perception of the examiner. Based on his knowledge and clinical experience, the patient's results may be interpreted differently.

Posturography testing systems that incorporate force platforms are considered to assess balance and postural control with greater sensitivity and objectivity than conventional clinical tests. The downside is their high cost which usually exceeds the budget of medical centres or individual clinicians involved in medical activity. For this reason, few centres are equipped with dedicated equipment for stance and walking evaluation.

The current article introduces ENTy, a Romanian original solution for the objective assessment of Romberg and stepping test. This device also allows data recording and automated data logging for re-evaluation and comparison of results over time.

\section{Material and Methods}

Fifty persons with normal balance and no known vestibular/inner ear or osteoarticular problems were tested with both vestibulo-spinal tests.

Assessment was performed clinically (physicians' empirical evaluation of the Romberg and stepping test) and with the ENTy medical device (Figure 2). The clinical evaluation was performed independently by two ENT physicians with experience in the field of vestibular pathology.
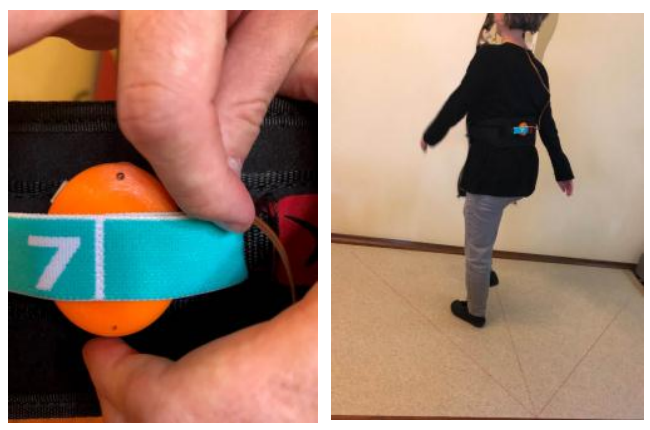

Figure 2. ENTy medical device

The base hardware ensures processing, communication and control, including power management optimisation and data pre-processing.

In order to have an easy to use solution, cluster and cable free, a wireless solution was deemed to be the best approach. Research by Hung and Zhang (2002) indicates that Bluetooth as a wireless communication technology is most appropriate for usage in medical environments, due to its low interference with medical equipment.

The device is based on the identification of the movements that are done through an inertial measurement unit (IMU). An inertial measurement unit is composed by an accelerometer, a gyroscope and a magnetometer. Besides the three sensors, there is also a digital signal processor (DSP) for data acquisitions from the three sensors and to apply a proprietary algorithm of fusion and filtration in order to obtain precise values about the linear acceleration, angular acceleration and the absolute angles of rotation on each axis.

The entire Graphical User Interface was built using JavaFX framework, and it provides functionalities for the doctor to manage patients and test protocols.

The graphical components are custom built, based on doctors' feedback, meeting the requirements of ENT specialists.

Figure 3 depicts the real time data visualisation tool provided by our implementation. 


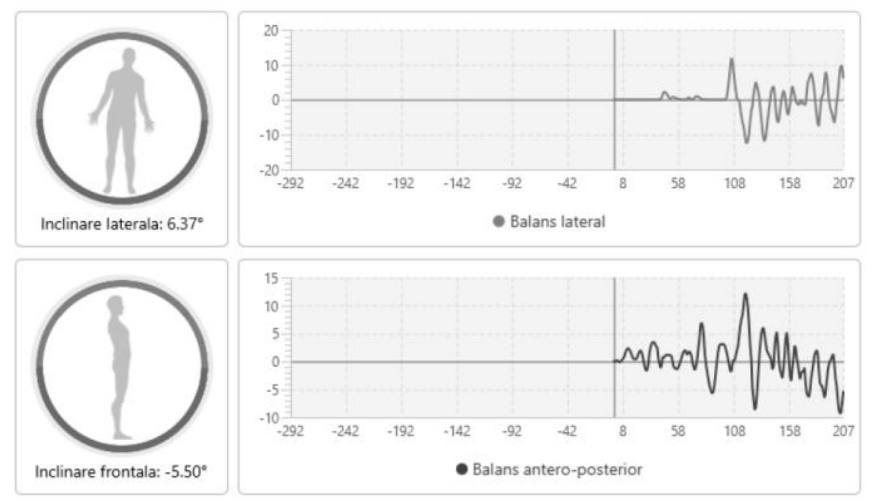

Figure 3. Real-time interface

After testing the application together with medical staff, the graphical representation of the final report for a given patient was adapted to match standard representations used in the medical field, with normal limits highlighted for easy at a glance interpretation (Figure 4).

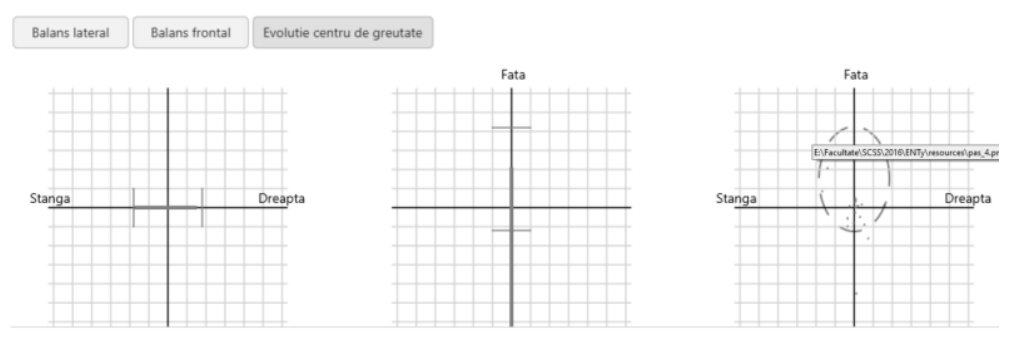

Figure 4. Final report - Graphical representation

\section{Results}

Currently, there is no equipment able to measure patient's responses in these two balance-test, based on the technology proposed by us.

The existing technologies involve large and very expensive equipment (force-plate platforms or walking evaluation systems) that can provide objective assessment of stance or stepping (Figures 5 and 6).

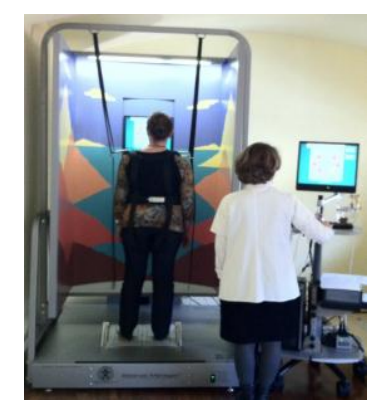

Figure 5. Pro Balance Master
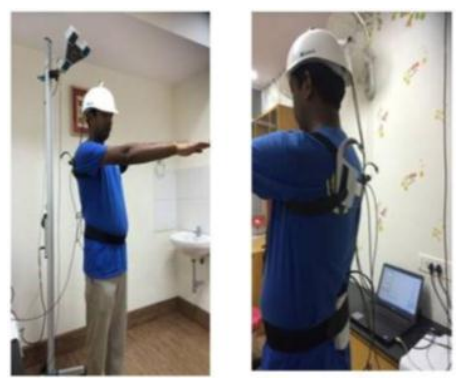

Figure 6. Ultrasound Craniocorpography system

Also, the focus of research in the last years is on new technologies for objective measurement of a patient's performance in the two vestibulo-spinal tests, but with different technologies. Dolinay, Pivnickova and Vasek (2014) used the skeleton tracking feature embedded on the Microsoft Kinect sensor in order to evaluate horizontal, vertical, angular and direction deviation. It covered only the Romberg test. Last year, the Kinect device was withdrawn from the market by Microsoft.

Pivnickova, Pivnickova, Dolinay and Vasek (2014) have developed a device based on a balance board, an accessory for the Wii game system, which can only evaluate the Romberg test. 
Our main objectives with the new ENTy system are to develop an objective, automated assessment for stance and stepping tests, with specific characteristics:

- A friendly, easy to use system;

- A wearable wireless device, affordable by most physicians;

- Hassle-free installation, requiring no special space, no mechanical anchors or specialised actions;

- An output format easy for interpretation and ready for integration within the medical records;

- Available statistics and long-term follow-up on a patient's evolution, both for medical progress tracking and for work related medical check-ups.

The Romberg test, initially proposed in the year 1840, consists in assessing the ability of a person to stand as still as possible for 20 seconds. The subject's feet are close together, its eyes shut, and both arms are stretched forward (Classic Romberg test, Figure 7a). If the result is considered normal (no significant swaying or toppling) (Lacour, Dutheil, Tighilet, Lopez, \& Borel, 2009), the difficulty of the test is increased by placing the feet one in front of the other in tandem or by executing the test while standing on one foot (sensitised Romberg test, Figure 7b). The test was designed to evaluate the function of the dorsal longitudinal medullary cords affected by tertiary syphilis. The swaying of the body requires additional spatial orientation information provided by the vestibular receptors. The importance of the vestibular information increases when a subject move with its eyes closed. Therefore, these tests that evaluate stance, especially when the eyes are closed, are useful for assessing vestibular function and balance.
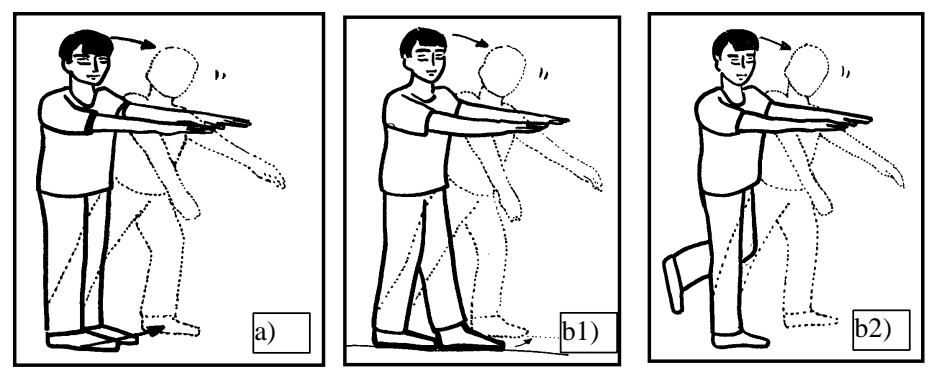

Figure 7. The Romberg test

a) Classic; b) sensitised (tandem - b1 and unipodal - b2)

Evaluating the functionality of the vestibular receptors, the central nervous system and the motor effector organs can be achieved dynamically through the test imagined by Unterberger and known as the Unterberger-Tretversuch test (1938). The patient is asked to walk in place with their eyes closed up to 50 steps (Fukuda test), either for 2 minutes (Unterberger test) (Figure 8).

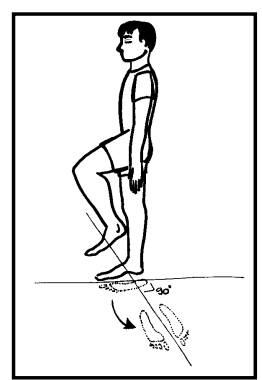

Figure 8. Unterberger stepping test

The examiner subjectively evaluates the subject's deviation from the initial starting position, either by the amplitude of the angle of deviation or by measuring the distance between the start and end points. Any deviation from the straight line greater than 50 degrees must be considered and taken into account with the rest of the clinical otologic and neurologic signs in order to establish a correct diagnosis (Bonanni \& Newton, 1998).

The most important directional mechanism during walking is responsible for maintaining the head's position so that the images perceived from our visual field remain still on the retina (otherwise dizziness occurs). For this 
reason, during balance testing, the positioning of the head compared to the trunk is also important. Because of this, the second sensor of the ENTy system is placed on the back of the head.

The centre of gravity (GC) is considered to be at 0.55 of the tested person's height (in front of the navel). It is considered to be that point within our bodies which, under the action of the same forces acting on the whole body, moves as if the whole weight of the body was concentrated at that level. For this reason, one of the sensors of the ENTy system is placed at the level of our centre of gravity.

The Base of Support (Figure 9) represents the area within the perimeter determined by the lines of contact between the subject's feet and the support surface.

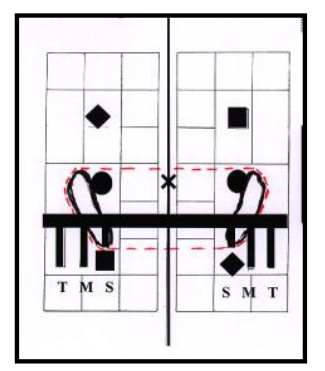

Figure 9. The base of support

Maintaining the vertical position of our bodies is a matter of maintaining or returning the centre of gravity of the body within the base of support without any external aid. In order for the test subject to perform this, it must withstand the destabilising influence of gravity or move its centre of gravity so that the centre projects inside the base of support at the end of the test. Because of this, one of the graphs obtained automatically after testing a subject with the ENTy system is the graph describing the swaying of the centre of gravity compared to normal swaying patterns.

The comparative clinical and paraclinical (ENTy) assessment of patient stability during stance, during the Romberg test and the ability to maintain the correct trajectory during the stepping test, revealed a good correlation between the subjective and objective test results. Testing was performed on a statistically significant group of 50 patients.

\section{Conclusion}

The Romanian ENTy innovative medical device promises a good acceptance in the medical community due to its wearable wireless characteristics, friendly interface and automated evaluation and comparison with the normal results. It is designed to add value to the medical evaluation due to its objective results which will allow better communication in between physicians and medical personnel. It provides an output format ready for integration with medical records, data recording and automated data logging with available statistics. These specifications allow long-term follow-up of a patient's evolution for both medical progress with specific therapeutic management tracking and work-related medical check-ups.

Future research is needed for validating the ENTy system into the objective battery test for patients with balance disturbances.

\section{References}

Abramovich, S., \& Prasher, D. K. (1986). Electrocochleography and brain-stem potentials in Ramsay-Hunt syndrome. Archives of Otolaryngology - Head and Neck Surgery, 112(9), 925-928. https://doi.org/10.1001/archotol.1986.03780090021002

Bonanni, M., \& Newton, R. (1998). Test-retest reliability of the Fukuda Stepping Test. Physiotherapy Research International, 3(1), 58-68. https://doi.org/10.1002/pri.122

Cnyrim, C. D., Newman-Toker, D., Karch, C., Brandt, T., \& Strupp, M. (2008). Bedside differentiation of vestibular neuritis from central "vestibular pseudoneuritis". Journal of Neurology, Neurosurgery \& Psychiatry, 79(4), 458-460. https://doi.org/10.1136/jnnp.2007.123596 
Dolinay, V., Pivnickova, L., \& Vasek, V. (2014). Objectivization of traditional otoneurological examination based on Kinect sensor Hautant's test based on Kinect. In Proceedings of the $201415^{\text {th }}$ International Carpathian Control Conference (ICCC) (pp. 91-94). https://doi.org/10.1109/CarpathianCC.2014.6843575

Georgescu, M. (2017). Vestibular rehabilitation - Recommended treatment for permanent unilateral vestibular loss. International Journal of Neurorehabilitation, 4(4). DOI: 10.4172/2376-0281.1000282

Georgescu, M., \& Stoian, S. (2011). Neuronita vestibulară în sarcină - prezentare de caz [Vestibular neuritis in pregnancy - Case presentation]. Ginecologia, 7(23), 58-61.

Georgescu, M., Stoian, S., Mogoanță, C., \& Ciubotaru, Gh. V. (2012). Vestibulary rehabilitation - election treatment method for compensating vestibular impairment. Romanian Journal of Morphology and Embryology, 53(3), 651-656. Retrieved from https://www.ncbi.nlm.nih.gov/pubmed/22990562

Hung, K., \& Zhang, Y.-T. (2002). Usage of Bluetooth in wireless sensors for tele-healthcare. EMBS/BMES Conference (Vol. 3, pp. 1881-1882). Houston, TX, USA. https://doi.org/10.1109/IEMBS.2002.1053074

Lacour, M., Dutheil, S., Tighilet, B., Lopez, C., \& Borel, L. (2009). Tell me your vestibular deficit, and I'll tell you how you'll compensate. Annals of the New York Academy of Sciences, 1164, 268-278. https://doi.org/10.1111/j.1749-6632.2008.03731.x

Neuhauser, H. K. (2007). Epidemiology of vertigo. Current Opinion in Neurology, 20(1), 40-46. https://doi.org/10.1097/WCO.0b013e328013f432

Pivnickova, L., Dolinay, V., \& Vasek, V. (2014). Evaluation of static posturography via the Wii Balance Board. In Proceedings of the $201415^{\text {th }}$ International Carpathian Control Conference (ICCC) (pp. 437-441). https://doi.org/10.1109/CarpathianCC.2014.6843643 